Itinéraires Itinéraires

Littérature, textes, cultures

2010-2 | 2010

Les blogs

\title{
Se donner un genre : pour une poétique du blog
}

\section{Christèle Couleau}

\section{OpenEdition}

\section{Journals}

Édition électronique

URL : http://journals.openedition.org/itineraires/2082

DOI : 10.4000/itineraires.2082

ISSN : 2427-920X

Éditeur

Pléiade

Édition imprimée

Date de publication : 1 juillet 2010

Pagination : 177-190

ISBN : 978-2-296-12012-9

ISSN : $2100-1340$

Référence électronique

Christèle Couleau, « Se donner un genre : pour une poétique du blog », Itinéraires [En ligne] 2010-2 | 2010, mis en ligne le 01 juillet 2010, consulté le 02 mai 2019. URL : http://

journals.openedition.org/itineraires/2082 ; DOI : 10.4000/itineraires.2082

\section{(c) (1) $\Theta$}

Itinéraires est mis à disposition selon les termes de la licence Creative Commons Attribution - Pas d'Utilisation Commerciale - Pas de Modification 4.0 International. 


\title{
Se donner un genre: pour une poétique du blog
}

\begin{abstract}
The present article deals with the poetical process allowing blog to be thought and constructed, from the inside, as a genre. Comparing blog with such other genres as diary and serial story, but also taking into account some characteristics peculiar to it - such as its interaction with the real reader, or the way to define the ethos of the author - draws the outlines of an endlessly renegotiated contract of reading. So, the establishment of blog as a genre appears to be the subject of an on-line collective elaborative process.
\end{abstract}

Keywords : blog, editorial genre, poetics, contract of reading, ethos

Mots clés : blog, genre éditorial, poétique, pacte de lecture, ethos

Pris entre un discours médiatique qui voudrait en faire le genre contemporain et des analyses littéraires et communicationnelles qui soulignent à la fois ce qu'il doit à des genres existants et son emprisonnement dans des contraintes formelles imposées de l'extérieur, le blog apparaît comme une formule moins stabilisée qu'il n'y paraît. L'hybridation générique, qu'il ne semble incarner qu'au prix de tensions internes, débouche moins sur un cahier des charges que sur un éventail de postures et d'attentes parfois contradictoires. Les impératifs techniques, le formatage qu'ils impliquent font l'objet de détournements et de négociations. Les chiffres de progression traduisent une pratique massive, désirable, mais en quête de légitimité.

Comment naissent les genres? Le baptême officiel est toujours tardif, la reconnaissance rétrospective. Par principe, l'évolution générique déplace les lignes, redéfinit les pondérations, redistribue les critères. S'inspirant de Wittgenstein dans une réflexion sur la théorie des genres, Antoine Compagnon a cette formule qui se charge de résonances particulières lorsqu'on l'applique à l'univers des blogs : 
Le concept de type est ici indispensable. Le type est un pont entre des occurrences (tokens), un lien entre l'unicité (le caractère privé) du sens et la sociabilité (le caractère public) de l'interprétation. Un sens a toujours des aspects uniques, mais il doit appartenir à un type pour être communiqué, pour être compris (et même pour être conçu). Le type comporte des implications, des traits (l'air de famille) ${ }^{1}$.

Faisceau de traits communs, expérimentés assez régulièrement pour créer une attente chez qui commence une lecture placée sous sa bannière, le genre ne rassemble pas qu'une famille d'énoncés : il institue par le même geste une communauté de lecteurs, dessinée en creux par sa configuration énonciative, et susceptible de matérialiser cette attente. L'ombre du genre place aussi le scripteur dans une réalité augmentée, subsumant son identité singulière - son univers, son style, son ton - dans une posture, un ethos légitimé.

Il s'agira donc moins ici de se demander si le blog est, ou non, un genre, que de chercher les indices montrant qu'il est vécu comme tel, et de mettre en lumière les moyens qu'il se donne pour se construire en tant que tel. Quand des auteurs prennent à bras le corps le genre auquel ils pensent appartenir, qu'ils se l'approprient, le font ou le défont, ils contribuent à le construire de l'intérieur. Qu'est-ce qui, dans leur pratique des soi-disant « lois du genre », est assumé, revendiqué, ou rejeté ? Qu'est-ce qui est déplacé, miné, réorienté?

Il est ainsi tentant de relire les blogs sous l'angle d'une micro-généricité, à la recherche de manifestations ténues du genre en deçà de la forme éditoriale téléguidée par les spécifications techniques de l'outil de blogging. Une généricité diffuse, en filigrane, comme à la marge, mais proposant une contractualisation au coup par coup, sur un mode additif et correctif à la fois. Pour reprendre une expression de Michel Serres, tout discours « dessine une place où se place celui qui tient à tenir ce discours ${ }^{2} »$. Cette démarche nous semble être au cœur du pacte de lecture des blogs.

\section{Lire les blogs ou la tentation de la continuité}

Nous parlerons de contrat de lecture, puisqu'on persiste à lire les blogs, alors qu'on consulte un site web. L'arborescence parfois complexe du site - qu'elle vise à organiser le cheminement dans l'information ou à égarer volontairement le lecteur, comme aime à le faire Philippe de Jonckheere - induit un parcours fragmenté. Pointilliste ou exhaustif, il découpe et réorganise l'ensemble écrit selon ses propres critères, une logique qui ne tient pas prioritairement compte du paramètre temporel. Le blog, en revanche, ne se départit jamais d'une forme de linéarité, qui projette nécessairement l'ordre de lecture sur l'ordre d'écriture, même si ces deux approches du texte ne se

1. Antoine Compagnon, «La notion de genre : 10. Genre et interprétation », Fabula, 4 mai 2001, http://www.fabula.org/compagnon/genre10.php, consulté le 19 avril 2010.

2. Michel Serres, Hermès III. La Traduction, Paris, Éditions de Minuit, 1974, p. 202. 
superposent pas forcément. La possibilité de lire les billets selon des regroupements thématiques établis par l'auteur, comme le caractère fluctuant du point d'entrée dans le blog, permettent, certes, des lectures transversales ou ponctuelles. Mais l'inscription de ces billets dans la continuité d'un ensemble chronologique invite le lecteur à prolonger sa lecture en ce sens. Arriver aléatoirement au beau milieu d'un blog parce qu'on a cliqué sur un lien n'est pas une expérience similaire à l'arrivée sur un site de presse, par exemple. Dans ce deuxième cas, il est fréquent de poursuivre son chemin dans une logique paradigmatique au moyen de nouveaux liens, ou de s'égayer sur des chemins de traverse par un pur hasard de contiguité. Liens et sérendipité sont présents aussi dans le blog, mais une troisième voie est offerte par l'immersion linéaire, qu'on peut interpréter comme un tropisme du narratif. Parallèlement, et souvent en introduction au modèle de lecture régulière assistée par flux RSS, la découverte du blog ouvre sur des pratiques de lecture intensive, le texte fragmenté se dévorant alors comme un bon roman :

et voilà!! $1 \mathrm{~h} 16$ du matin et je viens de finir de lire ton blog, à l'envers
siouplé $^{3}$

Ça y est! Après une semaine de lecture intempestive (j'ai découvert cet endroit début juin), j'ai fini de lire le blog (vachement très bien d'ailleurs) même si je savais déjà la fin. Je vais guetter les aventures d'après alors!!

Suivre l'intégralité du blog, ou le fil thématique qu'on a choisi, selon un ordre temporel, c'est recentrer le Web sur son axe syntagmatique - que celui-ci soit parcouru dans un sens ou dans l'autre. En aval, on veut connaître la suite, renouveler l'expérience, vérifier une adhésion ou un agacement, retrouver un même ton, un même esprit, exercés sur d'autres sujets. En amont, on cherche une cohérence, une explication : comment en est-on arrivé là? que signifie telle allusion? le billet est-il sérieux ou faut-il le lire au second degré? - bref, qui est ce blogueur, et d'où parle-t-il ? Sur le blog de Margaux Motin, on peut ainsi lire ce commentaire répondant, plus d'un an après, au premier billet du blog :

\footnotetext{
Haha c'est donc lààà que margoMotin a commencé :)

c'est attendrissant comment que tu savais pas encore que ça allait être du power bar :)
}

La posture inaugurale, la sincérité d'un premier billet éclairent après coup la connaissance qu'on a des pages les plus récentes, suscitant l'envie de reprendre à zéro le cheminement de lecture. Le blog est rattrapé par sa linéarité, qu'il s'agisse pour le lecteur de remonter aux origines d'une

3. Les pages citées peuvent être consultées via la version en ligne de cet article sur le site du CENEL, http://www.univ-paris13.fr/cenel/articles/CouleauBlogs.pdf. Orthographe et ponctuation originales sont respectées. 
success-story, ou pour l'auteur de revendiquer une cohérence d'ensemble. Le lecteur de Ma vie c'est de la merde se voit ainsi interpellé :

Maintenant, je ne doute pas que tu suis ce blog depuis un petit moment, et j'ose espérer que tu as lu les précédents textes que j'ai pondus! même les plus pourris comme ceux du début. Néanmoins, ils répondent tous à la même logique $[\ldots]$ sur n'importe quel sujet.

Le blog fait corps. Et ne pas exposer ouvertement la trame de sa vie n'interdit pas de souligner le fil de ses pensées. Si les journaux en ligne s'inscrivent évidemment dans ce rapport au temps, les chroniques les plus factuelles, au-delà de leur continuum thématique, tendent à se raccrocher aussi à la linéarité des travaux et des jours - fils personnels se mêlant aux considérations thématiques (« nous aussi on a une vraie vie » clament en sous-titre les participants à un blog collectif d'entreprise, Gameblog), allusions éclaircies ailleurs, effets de suite ou d'écho entre différents billets... Olivier Schmidt-Chevalier, qui joue des limites «méta » du genre dans Bloguer ou ne pas bloguer, s'amuse de ce phénomène :

Google Analytics : là, encore (eomme hier) pour la défunte v2.

Souligner le malgré soi de la continuité, c'est cependant s'y soumettre, à défaut de la revendiquer. Analysant au long cours ce qu'il entend, lit, observe, l'auteur évoque implicitement le contre-champ narratif de sa vie, et interroge celui de son lecteur. Mais surtout, l'établissement d'un compagnonnage avec le lecteur, réel ou supposé, finit toujours par créer un vécu commun, auquel l'auteur est amené à se référer. Plongé dans le blog, tout fragment s'ancre dans une continuité.

\section{La suite au prochain numéro ou la loi des séries}

Cette tension entre fragmentation et continuité n'est pas sans rappeler les logiques sérielles. Le blog, pris dans son ensemble, se rapproche en effet des séries éditoriales, son apparence technologiquement formatée, qui le rend immédiatement reconnaissable, l'inscrivant dans une même démarche de répétition/variation. Mais dans son fonctionnement quotidien, il revisite davantage les séries feuilletonnantes : même diffusion scandée, même possibilité d'appréhender séparément chaque unité, même valeur ajoutée de la continuité qui permet de saisir la trame d'ensemble et de décrypter les allusions.

Cette régularité, au-delà de son caractère segmentant (elle distingue blogueurs au long cours et dilettantes vite découragés), apparaît comme un point nodal de la contractualisation générique. Dès le premier billet les blogueurs annoncent leur désir de constance. À l'instar de Guillaume dans Ma vie c'est de la merde, ils soulignent ensuite leurs efforts pour tenir le rythme : 
occuper ton attention le plus régulièrement possible. [...] Je me suis tenu la promesse que ce blog ne fasse plus le mort, et angoisse donc à chaque fois de trouver de quoi le nourrir.

Philippe de Jonckheere qui, dans Le Bloc-notes du désordre, préfère pourtant gommer la présence du lecteur, réaffirme explicitement ce pacte tacite :

[...] le bloc-notes devrait rester une parution hebdomadaire d'articles quotidiens, mais, le sort s'acharne contre ce rythme hebdomadaire puisque mon ordinateur ne veut plus rien savoir $[\ldots]$.

Pas très coutumier de ce genre d'adresse aux lecteurs du bloc-notes, je demande tout de même que l'on soit un peu patient, que cela finira par revenir et qu'il y aura un rattrapage, je ne voudrais pas que vous manquiez un soupir de ma vie pleine de trous.

Dans un autre style, Shalima (Merci pour le chocolat) s'excuse du coup de canif donné au contrat :

Ouille, bon ben là, je suis prise en flag' de mauvaise conduite blogguesque... me le pardonnerez-vous?

Toute négociation apparait alors moins comme un véritable contournement que comme une manière de réaffirmer sa fidélité à la règle : prétéritions, pseudo-excuses humoristiques, déclarations provocatrices, billets inspirés par le manque d'inspiration et rubriques ad hoc constituent les motifs récurrents de cette permanente réactualisation du pacte de lecture. Certains blogueurs transforment même l'essai en faisant de ces bouche-trous des rendez-vous de premier ordre : le courrier des lecteurs de Guillaume ou de Tata Nath, par exemple, deviennent des rituels à forte teneur contractuelle et métatextuelle, au point que cette dernière, dans son Petit précis de grumeautique, met en garde les lecteurs trop avides : elle ne délivrera qu' " un conseil foireux par semaine (après c'est trop, faut pas abuser des bonnes choses) ».

La réitération rythme le temps du blog, mais ne doit pas lasser. Pour fidéliser le lecteur, il importe de se faire désirer. La présence au blog doit être continue mais non uniforme, s'agrémenter de variantes, d'effets de rythme, de suspens. Pétronille intitule ainsi un billet de son Beulogue:

« Où l'attente se fait insoutenable (et le suspense aussi)»

\section{Margaux Motin renchérit :}

Tu vas voir internaute, dans le prochain post, c'est plein d'été, d'amour et de sensualité ( $\mathrm{t}$ 'as vu cette accroche de ouf pour te faire revenir...)

Cette poétique de l'accroche peut s'exercer au second degré, ou chercher chez le lecteur des résonances plus profondes. Participant à l'expérience collaborative proposée par François Bon, Le Petit Journal (vos vies en deux lignes), Philippe de Jonckheere écrit ainsi : 
Nathan s'élancera-t-il ce soir du haut du toboggan de la piscine de Montreuil, la même question en suspens tous les jeudis soirs. La suite au prochain numéro.

Le retour du même, l'angoisse diffuse prennent corps dans le dispositif de mise en attente du blog - demain nous en saurons plus, plus que n'en sait l'auteur lui-même car dans sa lutte contre l'autisme la fin du feuilleton n'est pas encore écrite. Le suspens n'est pas jeu mais partage d'une même attente, invitation à un devenir.

\section{«I am the law ${ }^{4}$ "- donner sa juste place au lecteur}

Reconnaître une règle, la détourner ou l'habiter, ce n'est pas forcément y soumettre l'ensemble du processus - la fidélisation a ses effets pervers. L'auteur de Nom de code Sigmund a pu d'abord être flatté des cent trentehuit messages commentant l'annonce de la naissance de son fils. Pourtant, après les félicitations d'usage, très vite la question qui domine est celle de l'arrêt d'un blog dévolu aux «angoisses d'un futur père ». Le silence de l'auteur se prolongeant, la machine des commentaires tourne à vide et donne l'impression que le rapport de force s'inverse :

Oui, c'est ça l'emm...nuyeux avec les blogs à succès, c'est que les fans en demandent toujours plus...

Tu sais, Papa de Sigmund, il ne faut pas laisser la porte de ton blog ouverte comme ça à des inconnus. Des fois, les gens, ils entrent, ils s'y plaisent et puis ils s'installent.

[...] tu pourrais peut être juste créer une note supplémentaire ici, d'au moins deux lignes, histoire que nous puissions continuer à bavarder tranquillement dans les commentaires???

Si l'atmosphère reste bon enfant, et que le transfert de blog a lieu finalement sans heurts, il n'en reste pas moins que l'auteur a été un temps bousculé : harcelé, squatté, et pour finir quasiment expulsé de son propre univers, qui pourrait bien continuer à tourner sans lui ${ }^{5}$.

Bruno Devauchelle, revenant dans son blog sur son renoncement à animer une liste de diffusion, tient un discours très explicite à ce sujet :

Écrire un livre, c'est détenir une autorité, développer un site d'information aussi. Tenir un blog c'est proposer son autorité au lecteur, Créer une liste de diffusion, c'est prendre le risque d'une perte d'autorité. [...] L'autorité de l'auteur se trouve perdue dans les nouveaux modes d'usage des supports sur lesquels il s'exprime surtout s'il tente de donner la parole au lecteur.

4. Guillaume, Ma vie c'est de la merde.

5. Sur Le blog de vetetix, en revanche, le troll déclenché par un billet sur Mickael Jackson tourne à l'aigre. 
D'où chez certains blogueurs une réelle méfiance, qui peut aller jusqu'à la suppression volontaire des commentaires. Cette désactivation d'une des fonctions de l'outil apparaît comme une reprise en main du genre, dont la pratique redessine de fait les contours. Philippe de Jonckheere explique ainsi tenir compte des degrés d'investissement très différents qu'implique la rédaction des billets, " d'un ordre très personnel, intimiste ", ou coûtant « beaucoup d'efforts de recherche et de rédaction », et des commentaires, plutôt « de l'ordre du bavardage ». Surtout, ces réactions imprévisibles risquent de perturber à la source son projet d'écriture : « $\mathrm{Ce}$ ne serait pas très sain d'anticiper ces réactions, encore moins de les provoquer. »

Refuser ce qu'il désigne comme un « intégrisme » c'est clairement défendre sa propre conception du genre face aux poids conjugués de la technologie et de l'usage. Imposer silence au lecteur, non pour esquiver sa présence, fondatrice du pacte de lecture, mais pour ne pas dénaturer sa propre voix, telle semble être pour lui la condition d'une réelle autonomisation du texte littéraire ${ }^{6}$.

Kek semble partager cette analyse, bien qu'il y réponde autrement. La mise en ligne, au fil de l'écriture, de son roman graphique, Virginie, une histoire qui sent la colle Cléôpatre, suscite des commentaires de plus en plus nombreux. Cette invasion de l'espace de parole par le lecteur, qu'il n'interdit toujours pas dans son dernier blog bien qu'il tienne à la qualifier de « malsaine » sur le billet final du précédent, sera donc sans arrêt contrecarrée, limitée, contenue. Dans Bienvenue chez Kek, non content de décrédibiliser toute prise au sérieux des commentaires par une dénomination fantaisiste de leur décompte automatique ( 35 omoplates de lombrics polonais »), il prend le contre-pied des habituelles invitations à la prise de parole (« lâchez vos coms! » devient « faut vous calmer sur les coms »). Il reprend possession du lien permettant l'accès aux commentaires par l'ironique créativité qu'il y déploie :

23 commentaires ça commence à faire beaucoup je trouve

115 commentaires, partez maintenant. Allez chez Damned par exemple

54 commentaires auxquels je ne répondrai pas

Cette perpétuelle réaffirmation de la prééminence de l'auteur, seul maitre à bord, passe par des ruptures du pacte de lecture que ne renierait pas, dans l'intention, un Diderot. Kek poste ainsi un leurre, une fausse planche qui pervertit brutalement le cours des aventures de Virginie, puis s'esclaffe :

uh uh uh... regardez vos têtes... c'est juste parce qu'il y a trop de monde qui lit cette histoire, et je voulais vous emmerder un peu... Roh, ça va hein, on peut pu déconner. Mais pour lire la suite qui s'est réellement passée, cliquez là.

Contrairement à l'impression que peut parfois donner la possibilité de commenter les billets, la relation auteur/lecteur reste, dans les blogs,

6. Entretien à paraître en 2010 dans la revue Genesis, éditions de l'ITEM. 
profondément dissymétrique - on glisse sinon vers le forum. L'hospitalité a ses règles, le lecteur est toujours en résidence surveillée. Loin des expériences de création interactive, l'auteur seul mène le jeu, Guillaume tient à le rappeler :

[...] désolé de te décevoir sur ce point, inutile de me prier de faire «plus de ceci» ou «moins de cela», je fais ce qui me chante et j't'emmerde j't'embrasse bien fort.

Qu'il s'agisse d'ouvrir ou de fermer la porte du blog, de s'exposer au lecteur ou de prendre ses distances, de le séduire ou de s'en moquer, on observe un même désir d'orchestrer pleinement cette relation à géométrie variable.

Le blogueur, comme tout écrivain, a le souci de construire son lecteur. Mais alors que le livre configure cette relation virtuelle en amont, construisant un lecteur modèle ${ }^{7}$, le blog la contractualise plutôt en aval, par le biais de négociations et de réajustements avec le lecteur réel - et cette relation semble devoir être d'autant plus maîtrisée que l'interaction est effective. Les réactions de l'auteur aux commentaires ont de ce point de vue une grande importance, qu'il s'agisse de rappeler les grands principes du pacte de lecture, ou d'en édicter les règles les plus concrètes :

Attention, cet article est à prendre au second degré... car vu les commentaires d'internautes... [...] Merci de passer votre chemin si vous n'avez pas d'humour. (Maman presque organisée)

Tu as le droit de poster des commentaires ici, qu'ils soient de qualité ou non, qu'ils soient puérils ou non, qu'ils soient écrits en français ou non, mais tu n'as pas le droit d'insulter les gens. (Le blog de vetetix)

Si tu dis preum's je te pète ta tronche. (Bienvenue chez Kek)

La résistance à l'appauvrissement de l'échange - lecture réductrice, pseudo-dialogue - comme les réticences face aux automatismes lexicaux (langage SMS, gimmicks) témoignent d'une même volonté d'éviter une normalisation par le bas. En commentant métatextuellement les interventions de leurs lecteurs, ces blogueurs travaillent à configurer le genre de l'intérieur. Redresser les pratiques, proposer une orthodoxie garante des libertés, définir une forme ouverte là où certains voient une zone de non-droit, c'est promouvoir la généricité contre l'usage.

C'est aussi définir le blog comme un genre adressé. Les interpellations du lecteur, des plus discrètes aux plus bouffonnes, rappellent sans arrêt que ce texte se joue en public ${ }^{8}$, qu'il ne saurait se passer d'interaction, même si

7. Umberto Eco, Lector in fabula, Paris, Le Livre de poche, 1990, p. 61 sqq.

8. Les blogs "privés", inaccessibles sans mot de passe, constituent bien sûr un cas particulier de ce point de vue. 
celle-ci est circonscrite, voire réduite a minima. Comme une bouteille à la mer, il est écrit pour un lecteur réel, même s'il n'est pas sûr de l'atteindre. Le parcours d'Aphykit illustre toutes les nuances de cette dimension phatique, le lecteur y est attendu, provoqué, quitté, ou encore rappelé :

Je suis reviendue [...] j'aimerais que vous reviendassiez aussi. C'est vrai, je vous attends. J'ai rallumé le chauffage, aéré, dépoussiéré, refait le plein du frigo. Je suis prête.

Personne?

Personne.

It's safe, je vous assure. J'ai même décontaminé la boîte à spams [...]

Toujours personne?

Bon, alors je n'ai plus qu'à écrire un truc méga-intelligent et drôlissime.

Dur, dur [...] Ou mettre une photo racoleuse de mes pieds? Non plus?

Allez, s'il vous plaît.

Promis, si vous revenez j'arrête tout.

Cette scénographie contrastée s'avère toujours payante, glanant son lot de commentaires : de fausses sorties en rappels, c'est bien à la manifestation du lecteur réel que l'on assiste, sans qui l'écriture ne semble plus avoir de sens. La chambre noire de l'écriture semble s'être déplacée dans cet espace exposé, privé et public à la fois, que hantent « les gens de l'intérieur de l'ordinateur de maman », pour reprendre la formule de Piti Gasson. Nefisa, dans Le Mange-rêves, le dit autrement, cherchant désespérément à restaurer dans un moment d'écriture hors ligne ce sentiment d'être réellement connecté :

Et je pensais à toi. Oui, c'est un peu con de ressasser tout ça dans des moments pareils, quand justement je suis ton antithèse.

Et j'écrivais, j'écrivais, mon stylo courant à l'aveuglette, j'ai un trou dans la tête et il te ressemble, et sur un coin de la page je dessine ton profil que je n'ai jamais vu. Adieu ma carapace, adieu inutile fierté, adieu tout ça et en ce moment, adieu toi que d'habitude je voussoie.

Tu me manques.

Malgré la volonté parfois affichée d'écrire pour soi seul, le blogueur ne saurait oublier que la Toile n'est pas une page blanche : il pratique moins une écriture d'écran qu'une écriture de lien, de réseau.

\section{Marginalité de masse - un ethos paradoxal}

Si le blog est une scène, quel rôle y joue l'auteur? Quelle est cette « place » qu'il se donne à travers son discours? Dominique Maingueneau, à travers la notion $\mathrm{d}^{\text {" }}$ ethos "générique" ${ }^{9}$ " explique que les pratiques littéraires "adoptent le plus souvent l'ethos attaché aux genres dans lesquels elles s'investissent ». Jamais totalement figé, cet ethos peut apparaître

9. Dominique Maingueneau, Éléments de linguistique pour le texte littéraire, Paris, Bordas, 1990, p. 76. 
particulièrement malléable lorsque le genre est lui-même en voie d'évolution ou de formation. Comme le suggère Ruth Amossy :

Chaque genre de discours comporte une distribution préétablie des rôles qui détermine en partie l'image de soi du locuteur. Celui-ci peut cependant choisir plus ou moins librement sa «scénographie » ou scénario familier qui lui dicte sa posture ${ }^{10}$.

Lorsqu'elle a commencé à bloguer, Mother-at-last n'y est pas allée par quatre chemins :

Alors voilà, je vais venir conter mes derniers instants de non-mère sur un blog. Y'a pas de raison...

Les autres ils le font!

Pas de statut autorisé, pas de posture de savoir, une simple conformité définissant un ethos par défaut. Approche naïve? Ce point de vue est pourtant partagé par des blogueurs au long cours. Syven, qui anime trois blogs, dont un collectif, et un site de « bêta-lecture » collaborative, reprend à zéro l'un d'eux en ces termes :

\footnotetext{
J'ai bien envie de faire simple, sans complexe, pour me défouler. Juste raconter ce que je vis, ce que je vois. Pourquoi ne pas faire comme tout le monde?
}

Ne plus avoir à donner le change, être lu/aimé pour ce qu'on est... Syven modifie son profil et s'en explique in situ : « j’ai décidé de ranger ma présentation proprette pour me montrer un tantinet plus honnête. » Rengainant le dragon qui servait de bannière à son ancien blog - Les mondes de Syven - , elle le remplace par un fond textile sur lequel se détachent un ordinateur et quelques bobines de fil : assumant pleinement ses deux passions (l'écriture de fantasy... et la couture), elle rebaptise humoristiquement son site Syven, mondes et travaux.

Simplicité, sincérité vont de pair chez les blogueurs avertis avec la conscience d'un relatif anonymat malgré tout :

Internet, c'est le psychanalyste du pauvre. Pour la modique somme de votre connexion, il vous ouvre un tumulte silencieux où l'on peut s'égosiller sans crainte d'être vraiment entendu.

Nefisa n'est pas dupe, les voix se perdent dans le flux, repêchées seulement çà et là par quelques lecteurs de hasard, qui deviendront peut-être des fidèles, ou par le filet plus précis des blogrolls. Avant qu'une certaine notoriété ne fixe en quelque sorte la ligne d'écriture du blog, une grande liberté préside donc à sa rédaction. C'est ce qui tente Aphykit :

10. Ruth Amossy, "Ethos », dans Patrick Chareaudeau et Dominique Maingueneau, Dictionnaire d'analyse du discours, Paris, Seuil, 2002. 
Je vais donc faire de l'écriture clandestine, secrète, non déclarée. L'avantage, c'est que je peux écrire tout décousu, tout comme ça me vient, sans imaginer d'histoire, écrire des recettes de cuisine, raconter ma vie trépidante et passionnante (si, si, promis, je vous assure). Comme personne ne me lit, personne ne me fera la remarque.

La conscience d'écrire hors des réseaux classiques de publication induit chez les auteurs de blogs le sentiment de prendre le maquis. Pensant son fief, qui comme une bulle, qui comme un îlot de résistance, chaque blogueur, au fil des billets, affine son ethos par différenciation, scissiparité. Il n'a de cesse de se distinguer. D'où l'importance, quantitative et qualitative, des billets critiques et autres « coups de gueules », grands combats ou séditions minuscules. Qu'il s'agisse de parler politique, médias, jeux, cuisine ou éducation, le blogueur fait sécession. Il privilégie les réseaux d'information parallèle, snobe le tout-venant médiatique. Même lorsque sa prise de parole s'auréole d'une caution institutionnelle (« professionnel de la profession », spécialiste avéré), voire s'intègre à une stratégie de communication professionnelle (blogs d'entreprise), il a soin de préserver, au moins en apparence, sa liberté de ton et de jugement - il évite la langue de bois, parle des coulisses.

Mais la plupart du temps, l'ethos du blogueur est déconnecté de l'institution. La pseudonymie court-circuite le moi social et limite les phénomènes d'autocensure. Le blog permet alors d'oser une prise de parole, une posture, sans crainte de retombées immédiates (professionnelles, institutionnelles, relationnelles, familiales). Il autorise à briser publiquement la loi du silence, le conformisme social. À travers la pratique de cette « écriture clandestine, secrète, non déclarée ", l'ethos du blogueur a tendance à se décaler vers les marges ${ }^{11}$.

Le cas des «parents indignes » regroupe tous les ingrédients de cette posture - un terrain idéologiquement contrasté, un regard social contraignant, une langue de bois bien rodée :

Je vous parle à présent en direct de la bienpensitude, au cœur de la vie sociale des 1-6 ans et de leurs mères - pères - autres (rayer les mentions inutiles),

souligne Anphykit, dans une volonté de se démarquer de ce milieu qui la stigmatise, de se rayer, donc, de cette liste qu'elle rejette à son tour. " On n'est pas raccord », clament haut et fort les Mauvaises mères, confrontées aux représentations politiquement correctes de la maternité, tandis que Caroline Allard, dans les Chroniques d'une mère indigne, se dit « complètement en marge de l'image du parent respectable ».

La légitimation de cet ethos de contrebande passe par un pouvoir de dissonance et de démystification. C'est Papa de Sigmund jouant les Barbebleue (« Vous qui passez ici, pauvres malheureux, inconscients des recoins

11. Cette position à la marge n'est cependant en rien un gage de qualité dans la production effective, et peut produire des blogs et des commentaires éthiquement peu défendables. 
sordides de ce blog, voici les billets qu'ils vous faut éviter à tout prix »), l'avertissement de Ma tasse de thé ("Ames sensibles : malheureusement au cours de la rédaction de ce bloug $[$ sic], de nombreux aspects romantiques ont été sauvagement foulés aux pieds par la dure réalité ») ou encore Margaux Motin dénonçant «THE gros bobard que toutes les meufs qui ont déjà un enfant racontent à celles qui viennent d'avoir leur premier : tu vas voir, c'est que du bonheur... ». Quand les parents bloguent, les enfants trinquent, mais au-delà de leur forte teneur humoristique, ces blogs apparaissent comme une tentative pour récupérer une parole confisquée (par les spécialistes, les médias, les proches bien intentionnés), pour réinvestir par le discours une image de soi malmenée par la norme - il s'agit moins d'une écriture de soi que d'une réécriture. Le ton est essentiel. Les mésaventures du quotidien deviennent des épopées domestiques, les antihéros gardent la tête haute en toute circonstance. L'auto-ironie rend les aveux moins amers, la provocation panse les blessures narcissiques, d'autant que quelles que soient les éventuelles réactions des lecteurs, l'auteur - ici Margaux Motin - a toujours le dernier mot :

Pendant que poupette termine ses céréales devant Dora (télé le matin mère indigne blabla je sais ouais ta gueule) MOI JE SUIS AU TAQUET DEVANT L'ORDI.

Cet instantané de l'auteur au clavier définit, à sa manière, une écriture en lutte, arquée contre le discours dominant.

Mais surtout, 1'ethos ainsi construit permet d'opérer une translation, un pas de côté : sortir du cercle, pour mieux trouver dans l'hypocrite lecteur du blog son semblable, son frère. Cette culture du happy few, menacée mais pas encore ébranlée par la tentation d'accroître artificiellement sa popularité ${ }^{12}$, doit être envisagée sous un angle plus qualitatif que quantitatif. Il s'agit surtout de viser juste, d'attirer qui peut comprendre et apprécier - on pourrait plus justement parler d'affinités électives. Les blogrolls, les tags, les commentaires croisés témoignent de ces phénomènes de reconnaissance réciproque, même si elles laissent de côté le simple lecteur. Or, si la publicité a toujours une cible, si la télévision a la ménagère de moins de cinquante ans, les blogueurs semblent surtout avoir un repoussoir que Guillaume désigne ainsi :

On ne peut pas dire que j'emploie les bonnes méthodes pour séduire l'internaute de base.

L'éthique éditoriale se donne donc volontiers à lire en creux, comme à rebours des autres pratiques médiatiques. Elle s'inscrit dans une culture de niche. Il s'agit dès lors de donner au lecteur un sentiment d'unicité : à rebours du prêt-à-porter technique, on donnerait dans le sur-mesure. Quelle

12. Voir notamment les techniques conseillées par Technorati. 
que soit l'ambiance graphique choisie, elle dépasse le simple effet tuning, et vise une identité forte, non consensuelle. On pourrait penser que l'obstacle technique bloque cette recherche :

Voilà que je m'affranchis du carcan prémâché du blog de base. ah? non? bon ben je fais ce que je peux, hein [...] (« La Taie d'oreiller »)

Mais, de fait, cette originalité trouve à s'exprimer dans les marges prédéfinies du blog, qui encadrent et rythment la page principale, lui adjoignant un certain nombre d'outils et de rubriques. Cet espace imposé devient le lieu privilégié d'une écriture à contraintes qui en mine la stricte utilité pour en faire une affiche permanente de l'ethos éditorial (contrairement au texte des billets, il reste toujours présent sur la page). Nous avons évoqué, déjà, certains de ces jeux de détournements. Zoridae, dans «De la sexualité de l'araignée », colore très personnellement sa relation à ses fans en baptisant son espace fan art " Piqués de l'araignée ». Les fausses citations qui agrémentent aléatoirement la bannière d' "À bas les bébés 》 font un pied de nez au besoin de cautionnement qu'exprime l'existence d'une telle rubrique. Lorsqu'un commentaire humoristique accompagne en рор up chaque occupant de la blogroll, lorsqu'un billet est « commis » et non plus écrit, lorsqu'un commentaire devient un « coincoin » et que tout contact avec l'auteur suppose de cliquer sur le lien « Disez-nous », l'esprit de dérision s'impose évidemment. Cette originalité de détail, cette façon de s'insérer dans les failles, d'utiliser toute marge de manœuvre laissée par l'outil, apparaît encore comme une façon de noyauter le genre, de le faire évoluer de l'intérieur.

«Bloguons-nous? », « Bloguer ou ne pas bloguer? », « ce truc est-il encore un blog? »- ces questions, récurrentes sur les pages parcourues, donnent du blog l'image d'un genre éminemment réflexif. Certes un grand nombre de blogs semblent échapper à de tels cas de conscience, focalisés qu'ils sont sur une pratique plus immédiate, moins pensée. Cependant, les aléas de la vie du blogueur - rythme, confrontation directe avec les lecteurs ou avec leur absence, dérapages divers -, en amènent beaucoup, même ponctuellement, à jeter sur leur pratique un regard plus interrogateur. Surtout, cette pratique, saisie dans ses grandes lignes comme dans ses détails les plus ténus, témoigne d'une inscription dans un cadre générique plus ou moins accepté, ou aménagé. Le désir d'écrire, enfin, motivé tout à la fois par un certain conformisme et une volonté d'affranchissement, place vite l'auteur face aux paradoxes du genre.

Appuyée sur une observation empirique, notre approche poétique du genre blog fait donc ressortir quatre dominantes caractéristiques du contrat de lecture qu'il propose. Mais elles ne semblent pas devoir être toutes mises sur le même plan. L'esthétique de la continuité et la logique sérielle empruntent clairement aux genres existants : leur réinvestissement, comme le constant rappel de leurs règles par un insistant discours d'escorte, les mettent 
au premier plan sans changer fondamentalement leur nature. En revanche, les relations du « vieux couple » (l'expression est dans le Beulogue) que constituent l'auteur et le lecteur sont non seulement mises au centre du texte, mais vécues sur le mode d'une interaction réelle, qui en change véritablement la portée. Tout d'abord parce que ce dialogue effectif est constructif, dans le sens où chaque échange, y compris le plus insignifiant ou le plus glissant, peut être l'occasion de définir un peu plus l'architecture du genre - cette dimension métatextuelle passant par de constantes renégociations, reconfigurations de l'usage. Ensuite parce que la nature de l'ethos mis en place, légitimé à la marge et marqué par la dissidence, encourage chacun à prendre parti, et favorise un interventionnisme de lecteur. Ces deux caractéristiques permettent à l'ensemble des acteurs du blog d'expérimenter ce que lire/écrire veut dire, et de participer à l'émergence d'une forme de généricité collaborative, dans ces nouveaux salons littéraires dont le centre est partout et la circonférence nulle part.

Christèle Couleau

Université Paris $13-C E N E L$ 